\title{
Optimizing macromolecular tandem mass spectrometry of large non-covalent complexes using heavy collision gases
}

\author{
Kristina Lorenzen, Cees Versluis, Esther van Duijn, \\ Robert H.H. van den Heuvel, Albert J.R. Heck*
}

Department of Biomolecular Mass Spectrometry, Bijvoet Center for Biomolecular Research and Utrecht Institute for Pharmaceutical Sciences, Utrecht University, Sorbonnelaan 16, 3584 CA, Utrecht, The Netherlands

Received 2 February 2007; received in revised form 8 May 2007; accepted 1 June 2007

Available online 20 June 2007

\begin{abstract}
This paper is dedicated to Peter Roepstorff on the occasion of his 65th birthday. We like to thank him for his continuing enthusiastic support and his infectious passion for science especially for the "fun-experiments". This paper describes the results of such experiments.
\end{abstract}

\begin{abstract}
We evaluated and optimized conditions for tandem mass spectrometry on macromolecular protein complexes, using a modified Q-ToF instrument varying the gas pressure inside the hexapole collision cell, and studied the influence of using different collision gases, e.g. argon, krypton and xenon. These variations affected ion transmission through the instrument and dissociation efficiency In particular the high mass fragment ions were generated more readily and transmitted more efficiently using the heavier xenon as collision gas. We used the $800 \mathrm{kDa}$ GroEL 14 -mer chaperone machinery for these initial studies. Applying the optimized tandem mass spectrometry conditions, i.e. relatively high pressures and xenon as collision gas, we studied in detail the influence the binding of one and two gp5 substrate proteins had on the gas-phase dissociation of the chaperonin complex constructing break-down diagrams. We observed that the binding of substrate polypeptides had a significant effect on the gas-phase stability of the chaperonin complex, with the complex containing one and two substrate molecules being less susceptible towards dissociation than the substrate free GroEL. Interestingly, the GroEL:gp5 and GroEL:(gp5) ${ }_{2}$ complexes dissociate exclusively via the elimination of GroEL monomeric ions, indicating that both gp5 substrates are likely encapsulated inside the two cavities of, respectively, the cis and trans ring of the GroEL. From all data acquired, we conclude that the heavier xenon is the preferred collision gas for tandem mass spectrometry on very large macromolecular complexes.
\end{abstract}

(C) 2007 Published by Elsevier B.V.

Keywords: Tandem mass spectrometry; Protein complexes; Collissional cooling; Xenon; GroEL

\section{Introduction}

For structural biology, macromolecular electrospray ionization (ESI) mass spectrometry has become a key method to study protein complexes of increasing size and complexity [1-8] culminating recently in the analysis of intact endogenously expressed heterogeneous protein complexes, such as the in RNA degradation involved exosome complex $[9,10]$. The analysis of proteins and protein complexes by mass spectrometry (macro-

Abbreviations: Q-ToF m/z, mass-to-charge; GroEL, Escherichia coli chaperone complex; gp5, major capsid protein of bacteriophage P22

* Corresponding author. Tel.: +31 302536797; fax: +31 302518219 .

E-mail address: a.j.r.heck@uu.nl (A.J.R. Heck). molecular mass spectrometry) has become possible because of the relatively gentle ionization procedure ESI, which can retain non-covalent interactions in protein complexes [11]. The mass analysis of larger proteins and protein complexes is not a routine technique, since careful optimization of operating conditions of the mass spectrometer is required, which so far, was difficult to realize using commercially available instruments. We and others have described modifications made to commercially available mass spectrometers enabling mass spectrometry on macromolecular complexes [12-14]. One of the most important findings has been that the pressures in the first and second vacuum chambers have to be increased to analyze large protein complexes [12,15-19]. This effect is attributed to the collisional cooling and focusing of large ions in the ion guides and, therefore, improved transmission through the ion guides and ToF [18] . 
Although ions of macromolecular complexes attain a large number of charges in ESI [2], making their kinetic energy high, their velocity inside the mass spectrometer is relatively low, which makes them susceptible to off-axis drifting. This may be even more applicable for ions formed in highly energetic dissociation processes such as collision induced dissociation, whereby fragment ions are released with significant off-axis kinetic energies.

However, in recent years it has been shown that tandem mass spectrometry can be used to dissect in a sequential fashion even large protein complexes of a few hundreds of $\mathrm{kDa}$ in the gas-phase to identify building blocks, and potentially the topology, quaternary structure and stability of the complex [5,20-22]. In such tandem mass spectrometry experiments, precursor ions are selected on the basis of their $\mathrm{m} / \mathrm{z}$, ratio in the first quadrupole mass analyzer after which the selected ions are dissociated in a gas-filled collision cell. The subsequent ToF analyzer then allows mass measurement of the product ions. Although these procedures are alike for small molecules, the collisional activation of macromolecular complex ions is rather different. Ions of macromolecular complexes, such as the $800 \mathrm{kDa}$ GroEL chaperone machinery, may attain tens of charges (approximately 70 for the chaperone complex GroEL), and with the voltage over the hexapole collision cell reaching up to a maximum of $200 \mathrm{~V}$ this leads to laboratory frame collision energies in the order of $15,000 \mathrm{eV}$. Typically these macromolecular ions are subjected to collisions with relatively static, small argon atoms in the gas-filled hexapole collision cell. When such macromolecular ions with high translational energies undergo inelastic collisions with neutral rare gas atoms, part of the translational energy may be converted into internal energy, leading to decomposition. To simplify the description of such a process, one needs to consider the center-of-mass (com) framework instead of the laboratory reference frame. The maximum available energy for the transfer of kinetic energy to internal energy, in the first collision event, is the relative energy $\left(E_{\mathrm{com}}\right)$ and depends on the collision partners' masses. Conversion from laboratory to center-of-mass collision energies is given by [23]:

$E_{\mathrm{com}}=\left(\frac{m_{\mathrm{t}}}{\left(m_{\mathrm{p}}+m_{\mathrm{t}}\right)}\right) E_{\text {lab }}$

where $E_{\mathrm{lab}}$ is the ion's kinetic energy and $m_{\mathrm{t}}$ and $m_{\mathrm{p}}$ represent the masses for the neutral target gas and precursor ion (800 kDa for GroEL), respectively. Using argon as a collision partner in this equation, it is evident that $15,000 \mathrm{eV}$ in the laboratory framework reduces to approximately $0.75 \mathrm{eV}$ in the center-of-mass framework, hardly sufficient to break any chemical bond. Therefore, it is generally expected that successful macromolecular tandem mass spectrometry depends on multiple collision events, as happening in the hexapole collision cell of the Q-ToF mass spectrometer. Considering this simple equation it is evident that with a heavier collision partner such as krypton or xenon considerably more energy may be transferred into internal energy. In this study, we report the use of the collision gases argon, krypton and xenon for macromolecular tandem mass spectrometry. We show that the pressure in the collision cell has a significant effect on the transmission of precursor and fragment ions, with in particular the lowly charged high mass macromolecular fragment ions being negatively discriminated at lower gas pressures. Additionally, we show that the heavier target gases result in more efficient fragmentation and fragment ion detection, making xenon the collision gas of choice in macromolecular mass spectrometry.

\section{Experimental}

\subsection{Chemicals and preparation of proteins}

GroEL was overexpressed in Escherichia coli strain MC1009 containing plasmid pSL6. Cells were grown in Luria-Bertani (LB) medium containing $100 \mathrm{mg} / \mathrm{l}$ ampicillin and $0.0005(\mathrm{w} / \mathrm{v})$ arabinose at $37^{\circ} \mathrm{C}$ under vigorous aeration. GroEL was purified according to a previously described protocol, slightly modified by the introduction of an acetone precipitation step [3,22]. Purified gp5 was a kind gift of $\mathrm{C}$. Teschke (University of Connecticut, USA).

The buffer was exchanged to $50 \mathrm{mM}$ ammonium acetate with a $\mathrm{pH}$ of 6.8 using ultra centrifugation filters with a cut-off of $10 \mathrm{kDa}$ (Millipore, Bedford, UK) GroEL gp5 complexes were formed by first unfolding the gp5 in $8 \mathrm{M}$ urea for $30 \mathrm{~min}$ at room temperature at a final gp 5 concentration of $25 \mu \mathrm{M}$. The unfolded substrate was added to $50 \mathrm{mM}$ ammonium acetate buffer containing $28 \mu \mathrm{M}$ monomeric GroEL at ratios varying from 1:0 to $1: 5$. The resulting maximum concentration of $1.7 \mathrm{M}$ urea did not affect the oligomeric GroEL structures. Excess of urea was removed from the sample by filtration while changing the buffer to $50 \mathrm{mM}$ ammonium acetate. Final concentrations of the GroEL proteins on the basis of their monomeric masses were $6 \mu \mathrm{M}$. Cesium iodide, used for calibration of the mass spectrometer, and ammonium acetate were purchased from Sigma (Zwijndrecht, NL).

\subsection{Mass spectrometry}

All the mass spectrometry measurements were performed in positive ion mode. Proteins and protein complexes were introduced into the mass spectrometer by using borosilicate needles in the absence of any backpressure. The needles were pulled from borosilicate glass capillaries (Kwik-Fil, World Precision Instruments, Sarasota, FL) on a P-97 puller (Sutter Instruments, Novato, CA), coated with a thin gold layer by using an Edwards Scancoat (Edwards Laboratories, Milpitas, CA) six Pirani 501 sputter. All the mass spectra were calibrated using cesium iodide (5-25 mg/ml) in 50\% (v/v) 2-propanol. The instrument used was a modified first generation Q-ToF 1 (Waters, Manchester, UK) equipped with a Z-spray source. Except where specifically indicated, all modifications to this instrument have been described previously [14]. A schematic diagram of the instrument is shown in Fig. 1. Electrospray ions are produced at atmospheric pressure after which the partly solvated ions are introduced into the first vacuum stage (P1) of the mass spectrometer through 
a sample cone with an orifice of $400 \mu \mathrm{m}$. The pressure in P1 was increased by reducing the pumping efficiency of the rotary pump (10 mbar). The molecular beam that evolves behind the sample cone is then extracted orthogonally through an extractor cone with an orifice of $1 \mathrm{~mm}$ into the second pumping stage (P2; $\left.1 \times 10^{-2} \mathrm{mbar}\right)$. The cone voltage used was $175 \mathrm{~V}$. Ions entering the second pumping stage (P2) were focused by a hexapole ion guide. The third vacuum chamber contains a quadrupole, a separate collision cell and a transport hexapole lens. The quadrupole in the modified Q-ToF had a frequency of operation of $300 \mathrm{kHz}$. This sets the theoretical accessible transmission limit to $32,000 \mathrm{~m} / \mathrm{z}$. When acquiring ToF spectra the quadrupole operates in RF-only mode as a broad-band mass filter, however, in the tandem MS mode the quadrupole transmits only ions in a narrow $\mathrm{m} / \mathrm{z}$ window around the set $\mathrm{m} / \mathrm{z}$, thus, operating as a narrow-band mass filter. Ions are then transferred to the hexapole collision cell, which was modified such that the collision gas pressure can be increased. The entrance and exit orifices of collision cell were varied between 1.5 and $2.0 \mathrm{~mm}$, respectively, as described in detail in the results section. The voltage over the collision cell was varied between 0 and $200 \mathrm{~V}$. To measure protein complexes we used a cylinder head pressure of $1.8 \mathrm{mbar}$ admitted to the collision cell via a needle leak valve with a $100 \mu \mathrm{m}$ capillary with a length of $1.25 \mathrm{~m}$ leading to a typical P4 pressure of around $2 \times 10^{-2}$ mbar. Using argon the resulting pressure at P3 was $6.7 \times 10^{-4} \mathrm{mbar}$ in case of the $2 \mathrm{~mm}$ orifices and $1.5 \times 10^{-4} \mathrm{mbar}$ in case of the $1.5 \mathrm{~mm}$ orifices.

\section{Results and discussion}

\subsection{Ion transmission and gas-phase dissociation of GroEL chaperone complexes using different collision gases and different entrance and exit orifices on the collision cell}

To analyze intact ions in vacuo from large complexes in solution conditions have to be optimized. Insufficient axial cooling makes ions miss the detector, whereas insufficient radial cooling results in poor transmission of ions through the different apertures in the mass spectrometer. We and others have noticed that increasing the collision gas pressure in the collision cell supports dissociation and ion transmission of high $\mathrm{m} / \mathrm{z}$ ions. The entrance and exit orifices of the hexapole collision cell (see Fig. 1 for their location) in our instrument have standard apertures of $2.0 \mathrm{~mm}$. When using argon and increasing the P4 pressure above $1.5 \times 10^{-2} \mathrm{mbar}$ the transmission of the ions decreased strongly (the signal was $20 \%$ lower when changing from 1.5 to $2 \times 10^{-2} \mathrm{mbar}$ ). The pressure in the $\mathrm{P} 3$ region increased to approximately $6.7 \times 10^{-4}$ mbar, which is at the limit of being tolerated by the instrument. As we hypothesized that increasing the collision cell gas pressure might further assist the dissociation and ion transmission, we evaluated smaller entrance and exit orifices of $1.5 \mathrm{~mm}$ as the gas leakage out of the cell is expected to decrease using these smaller apertures allowing us to achieve a higher pressure (P4) in the collision cell. We first tested whether ion transmission was affected using the different orifices and noticed decreases in total ion transmission in the order of $25 \%$

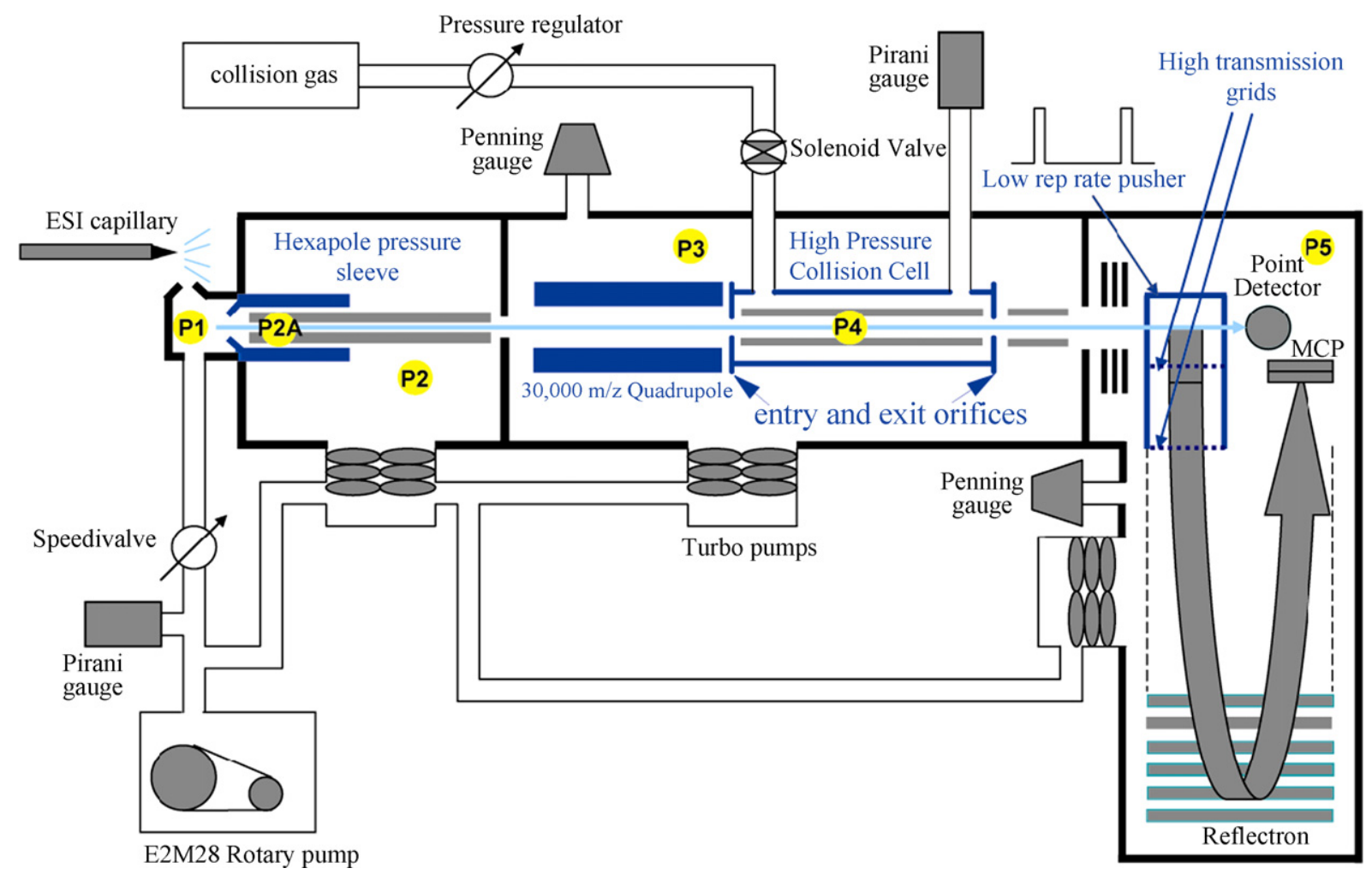

Fig. 1. Schematic layout of the modified Q-ToF 1 mass spectrometer. Details of most of the modifications have been described previously [14]. The position of the entrance and exit orifices of the hexapole collision cell, which sizes were varied in the current experiments, are indicated as well as the pressure read-outs mentioned in the manuscript. 
for ions with $\mathrm{m} / \mathrm{z}$ values around 10,000 , using the smallest apertures, a loss we took for granted. Using the smaller apertures of $1.5 \mathrm{~mm}$, we noticed that the collision gas pressure P4 could even be increased close to $3 \times 10^{-2}$ mbar (using argon). Now a $\mathrm{P} 4$ pressure in the collision cell of $2 \times 10^{-2}$ mbar resulted in a pressure $\mathrm{P} 3$ of $1.2 \times 10^{-4}$ mbar. Resulting in a decrease of the leakage effect of gas from the collision cell into the quadruople region which is six times lower with the $1.5 \mathrm{~mm}$ orifices than with the $2 \mathrm{~mm}$ ones.

Next we evaluated what the effect of increasing the collision cell pressure (using argon) had on the tandem mass spectrometry spectra of the 14-mer GroEL chaperone machinery. For these measurements GroEL complexes in $50 \mathrm{mM}$ ammonium acetate $\mathrm{pH} 6.8$, were used at a monomer concentration of $6 \mu \mathrm{M}$. The capillary voltage was typically set at $1500 \mathrm{~V}$, the cone voltage at $175 \mathrm{~V}$ and the ion energy at $2.0 \mathrm{~V}$. Produced ions were mass isolated in the quadrupole analyzer and accelerated into the target gas-filled collision cell. In Fig. 2 the tandem mass spectra are shown of the mass selected $69^{+}$ion of GroEL (at an $\mathrm{m} / \mathrm{z}$ value of 11,640 ) measured under identical conditions, with the collision voltage at the maximum of $200 \mathrm{~V}$. The argon collision gas pressure was varied from $1 \times 10^{-2}, 1.5 \times 10^{-2}$, $2 \times 10^{-2}$ to $3 \times 10^{-2}$ mbar. In all these cases, an equal amount of mass spectra were averaged. In Fig. 2, the spectra are normalized on the intensities of the monomeric fragment ions. The mechanism of the dissociation of subunits from protein com- plexes in tandem mass spectrometry is still a relatively poorly understood process, however, it is generally accepted that multicomponent protein complexes dissociate via similar pathways, i.e. expulsion of the smallest monomeric subunit [2] attaining an inequitable high amount of the charges. Recently, some interesting exceptions have been reported notably with surface induced dissociation [24], but also with specific "dimer-ofdimers" tetramers [14]. The gas-phase dissociation pathway of unliganded GroEL (800 kDa) has been well documented [20,22] and follows the generally observed trends described above. Upon isolation of the $69^{+}$ion of GroEL at an $\mathrm{m} / \mathrm{z}$ value of 11,640 a monomeric GroEL subunit becomes ejected from the tetradecameric complex. The monomers attain during the dissociation a large amount of the charges and are observed around $\mathrm{m} / \mathrm{z} 2000$ $\left(28^{+}\right)$, whereas the tridecameric ions are observed around $\mathrm{m} / \mathrm{z}$ $18,000\left(41^{+}\right)$. At higher collision gas pressure, a second GroEL monomer is dissociated from the tridecameric complex, resulting in the formation of dodecameric GroEL product ions. The dodecameric GroEL obtains an average charge state of only $20^{+}$. Therefore, these ions appear at a high $\mathrm{m} / \mathrm{z}$ values above $\sim 30,000$. The spectra shown in Fig. 2 reveals the effect of increasing the collision gas pressure. In the range studied, the total detected ion intensities of the monomer fragment ions were only changing about $10 \%$ with increasing pressure. Therefore, we used these ions to normalize the spectra. The higher $\mathrm{m} / \mathrm{z}$ ions show a significant increase in transmission. The ion intensities of the

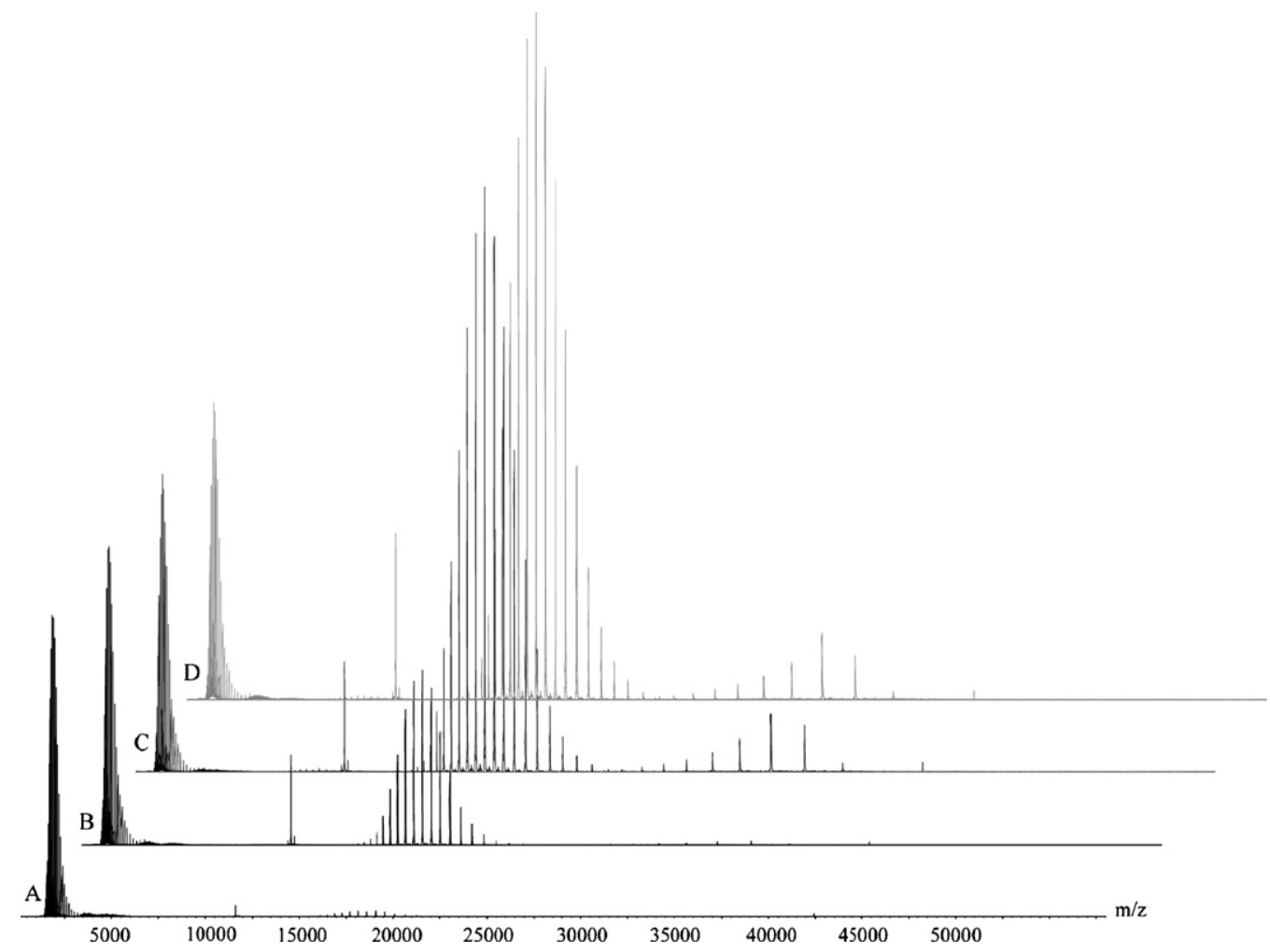

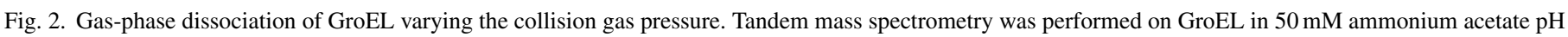

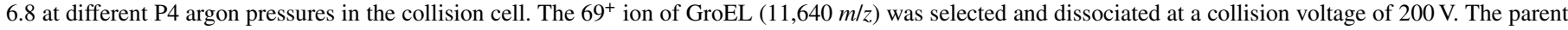

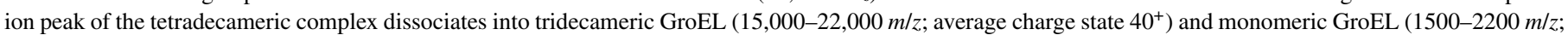

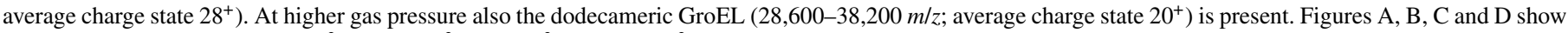
collision gas pressures of $1 \times 10^{-2}, 1.5 \times 10^{-2}, 2 \times 10^{-2}$ and $3 \times 10^{-2}$ mbar, respectively. 
high mass ions rise with increasing gas pressure, the precursor ions increases 1.5 times, the tridecameric fragment ions 4 times and the dodecameric fragment ions 10 times comparing the collision cell pressures of $1.5 \times 10^{-2}$ and $3 \times 10^{-2}$ mbar. In the ideal case, the summed intensity of the high mass fragment ions should be identical to the summed monomeric fragment ion intensities, which seems to be the case from a gas pressure of $2 \times 10^{-2} \mathrm{mbar}$ and upwards in the collision cell. We attribute the observed positive influence of increasing the gas pressure only marginally to increased dissociation of the precursor ions, but mainly too the improved transmission of the high $m / z$ precursor and fragment ions. We evaluated additionally a collision gas pressure in the hexapole of $6 \times 10^{-3} \mathrm{mbar}$, but then only monomeric fragment ions were observed, at an ion intensity reduced to $30 \%$, compared to the measurements at $1 \times 10^{-2}$ mbar. Therefore, reducing the gas pressure below $1 \times 10^{-2}$ mbar deteriorated the ion transmission too much. We tested the ion transmission also for krypton and xenon. While for krypton the presence of monomeric to oligomeric ions is equal starting at a pressure of $5 \times 10^{-3} \mathrm{mbar}$, xenon showed the same transmission efficiency from $1 \times 10^{-3}$ to $2 \times 10^{-2} \mathrm{mbar}$. Thus observed effects are very significant with the commonly used argon as collision gas, but they seem to diminish when using krypton and especially xenon. As the results with the aperture of $1.5 \mathrm{~mm}$ seem to be optimal for macromolecular mass spectrometry, we performed all later described experiments with these entrance and exit orifices.

\subsection{Energy resolved gas-phase dissociation of GroEL chaperone complexes using argon, krypton and xenon collision gases}

Following the optimization of the collision cell entrance and exit apertures, we investigated the effect of the identity of the collision gas. It is well known that the identity of the target gas may influence the dissociation efficiency, although such data is largely limited to the analysis of relatively small molecules/peptides $[23,25,26]$. Using identical experimental conditions, selecting the same precursor ions of GroEL, we performed tandem mass spectrometry using sequentially argon (average mass $40 \mathrm{Da}$ ), krypton (average mass $83.8 \mathrm{Da}$ ) and xenon (average mass $131.3 \mathrm{Da}$ ) as collision gas. For illustrative purposes we have depicted in Fig. 3 tandem mass spectra of mass selected $69^{+}$ions of GroEL measured under identical conditions, with the collision voltage at the maximum of $200 \mathrm{~V}$, only varying the nature of the collision gas between argon, krypton and xenon (bottom to top in Fig. 3). With all these three collision gases we recorded spectra with varying collision voltages, and calculated the ion intensities by averaging over all charge states of a particular ionic species. These ion intensities do evidently not correspond directly to species abundance, as several instrument response factors (transmission and detection) have to be taken into account. Here we just use the integrated ion intensities for relative comparisons. In Fig. 4 A, B and C these "break-down" diagrams are depicted, plotting the measured ion intensities of the GroEL precursor ions, the monomeric, the tridecameric and dodecameric fragment ions versus the collision voltage for argon, krypton and xenon as collision gases, respectively. In all these graphs a gradual relative decrease in precursor ion intensity is observed (the solid circles), although the onset of this decrease varies with the identity of the collision gas. Interestingly, using xenon and krypton the summed ion intensities of the concomitant fragment ions (i.e. monomeric versus tridecameric fragment ions) seems to be in line. This might be expected as for each monomeric fragment ion one tridecameric fragment ion is formed, indicating that the other instrument parameters influencing the response factors for these two very different ions are quite identical. In contrast, using argon as collision gas the ratio monomer versus tridecameric fragment ions is very much in favor of the monomeric ions, revealing that using argon the transmission of the tridecameric fragment ions is reduced, just as described in the previous section. To determine a relative threshold for dissociation we plotted in Fig. 5A the ratio (precursor ions/(precursor ions + monomer

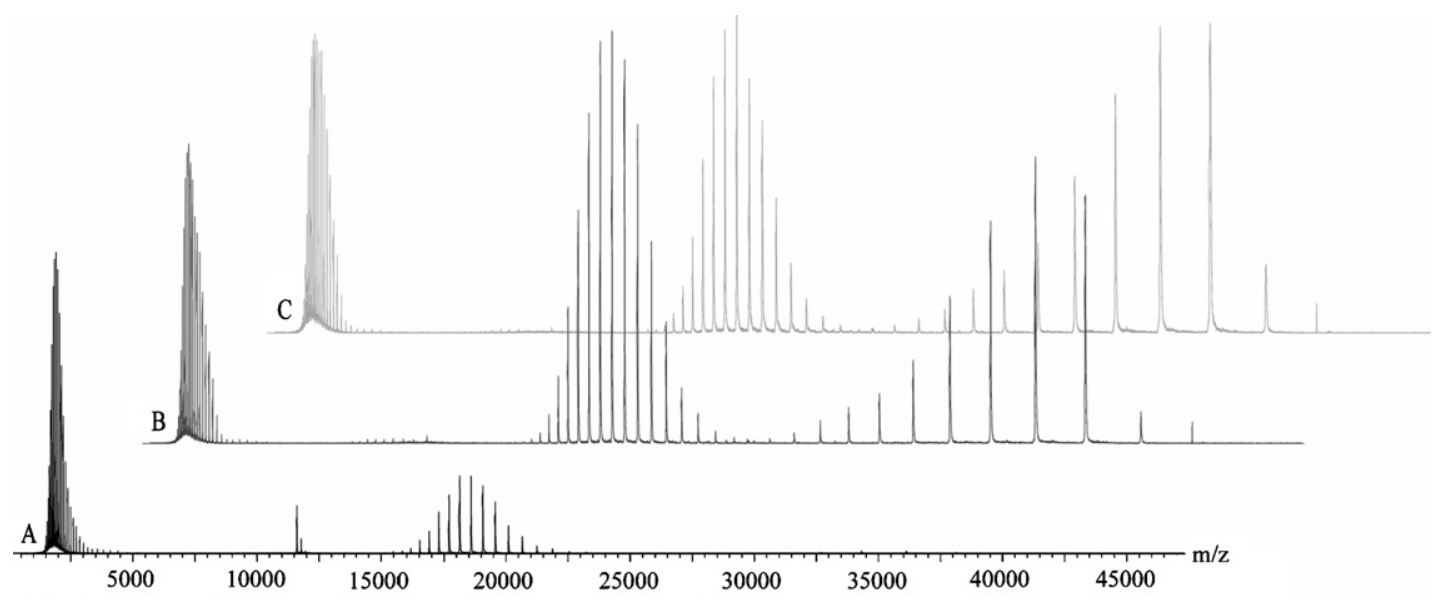

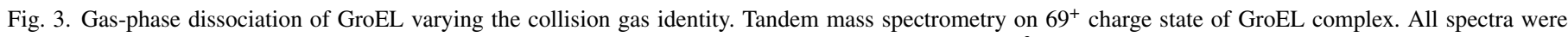

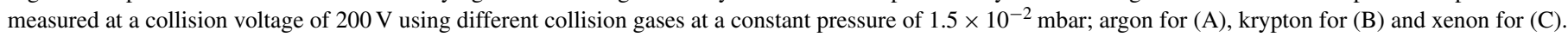

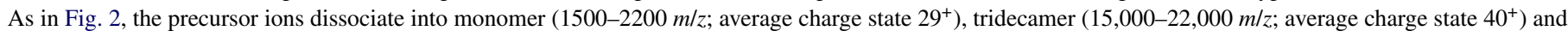
dodecamer $\left(28,600-38,200 \mathrm{~m} / z\right.$; average charge state $\left.20^{+}\right)$. 

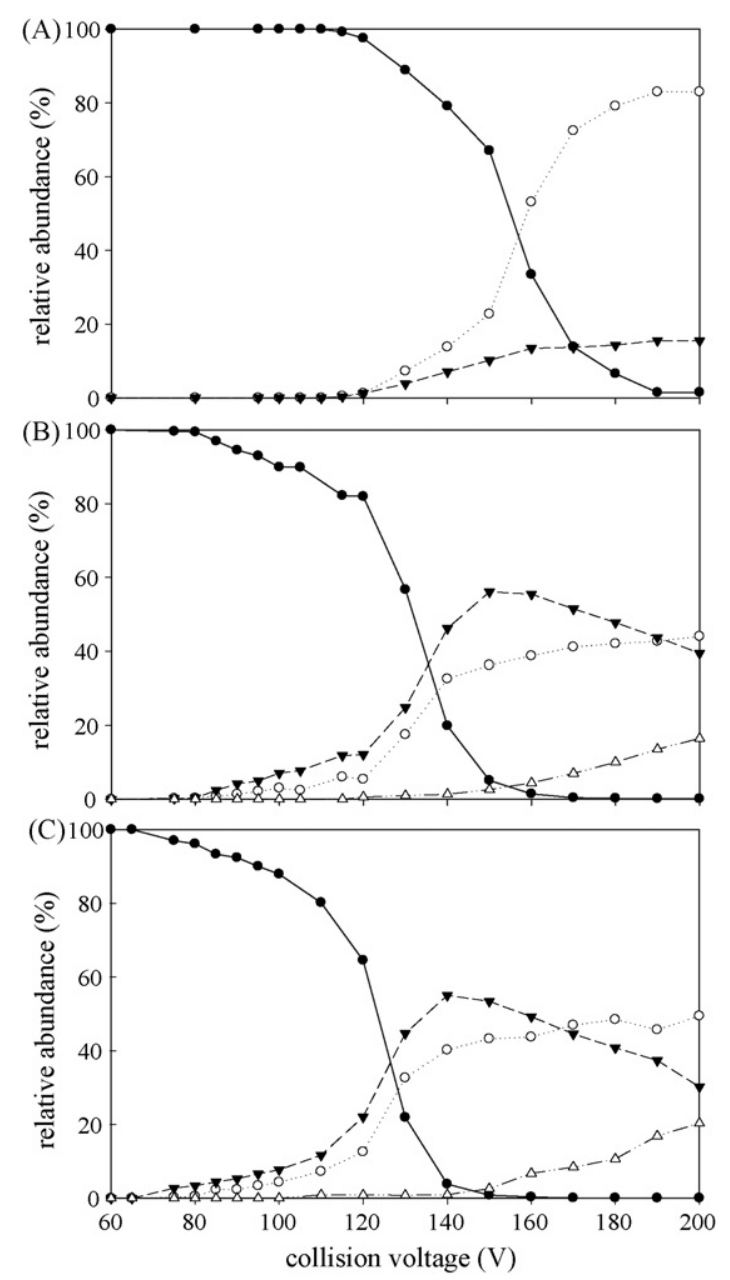

Fig. 4. Break-down graphs of GroEL using different collision gases. Ion intensities of the GroEL monomers, tetradecamers, tridecamers and dodecamers at increasing collision energies for argon (A), krypton (B) and xenon (C). The different ion intensities were integrated and plotted in relative abundance to the total ion intensity. The pressure of the collision gas was $1.5 \times 10^{-2} \mathrm{mbar}$ for all measurements; $(\bullet)$ represent the tetradecamer selected for dissociation $\left(69^{+}\right.$ at $11,640 \mathrm{~m} / \mathrm{z}),(\bigcirc)$ the dissociated monomer, $(\mathbf{\nabla})$ the tridcamer and $(\nabla)$ the dodecameric GroEL complexes.

ions) versus the collision voltage. These graphs reveal that the collision voltages at which the precursor ion intensity is reduced to $50 \%$ are approximately at 157,137 and $128 \mathrm{~V}$, for argon, krypton and xenon, respectively. In Fig. 5B these results are re-plotted, but now versus the center-of-mass collision energy. Evidently, in this graph the three curves should overlay, when the simple relationship Eq. (1) would be valid for our measurements. However, this seems not to be the case, indicating that Eq. (1) either overestimates the amount of collision energy converted into internal energy using argon, or underestimates the amount of collision energy converted into internal energy using xenon. Clearly, xenon is found to be a much more efficient collision gas in our measurements. The fact that the simple Eq. (1) is not valid may be due to the fact that it describes single collision events, whereas the collisional activation leading to dissociation of the macromolecular complexes described here is a multiple collision event, estimated to involve hundreds of collisions. This process is evidently much more efficient in case of xenon as
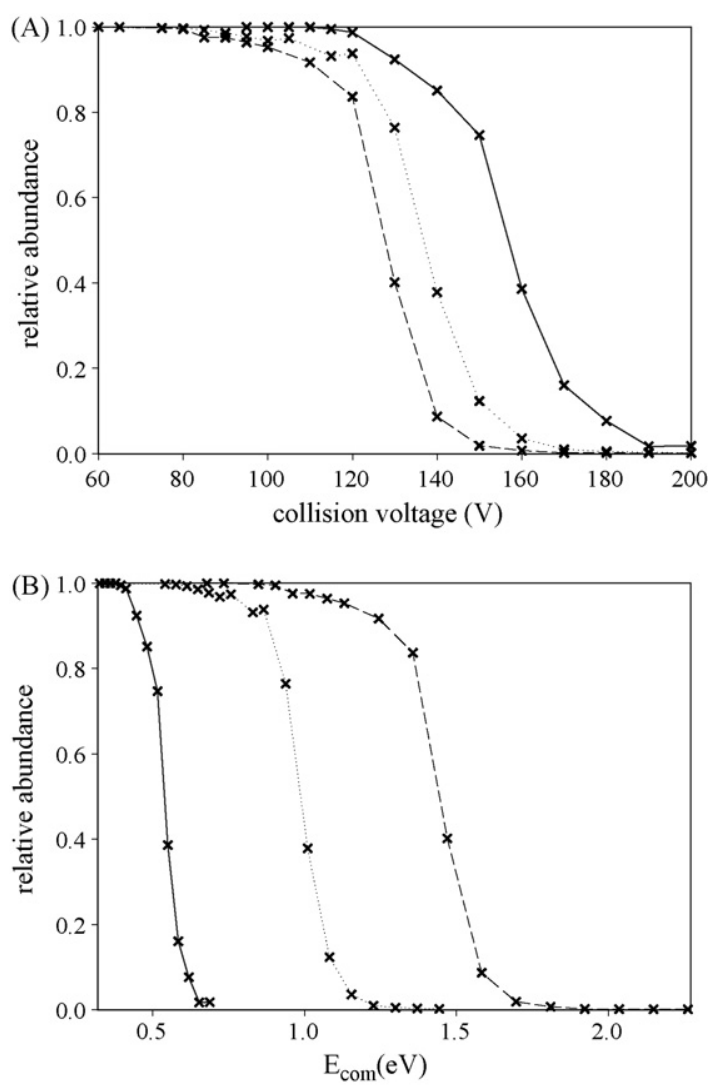

Fig. 5. Break-down graphs of GroEL precursor ions using different collision gases in the laboratory and center-of-mass frame. To construct relative breakdown curves of the GroEL precursor ions the ion intensity of the precursor was divided by the summed ion intensities of the precursor and monomeric fragment ions $(Y$-axis). Dissociation threshold scheme of the tetradecameric GroEL complex. These ratios are plotted vs. collision voltage in (A) and vs. center-of-mass collision energy $\left(E_{\mathrm{com}}\right)$ in $(\mathrm{B})$. The lines represent the different gases: - for argon, ... for krypton and - - - for xenon.

collision gas compared to argon. Previously, Mauk et al. [27] proposed a more elaborate model for the energy transfer from kinetic to internal energy in multiple collision events based on their experimental work on the dissociation of cytochrome $\mathrm{c}$. As we do, they also observed that the conversion to the center-ofmass collision energy as given in Eq. (1) does not fully make up for the conversion into internal energy. An argument for the more efficient dissociation observed with heavier collision gasses put forward by Mauk et al. is that the collisions with the heavier gas molecules slows the macromolecular ions more down, extending their residence time in the collision cell, giving them more time to fragment, an effect closely resembling the kinetic shift effect.

\subsection{Energy resolved gas-phase dissociation of GroEL, GroEL:gp5 and GroEL:gp5 $5_{2}$ chaperone-substrate complexes using xenon as collision gas}

In chaperone assisted folding, an unfolded polypeptide substrate is encapsulated in one of the rings of the GroEL chaperone. Binding of a substrate to one of the GroEL rings is generally assumed to have an anti-cooperative effect, i.e. binding of the 


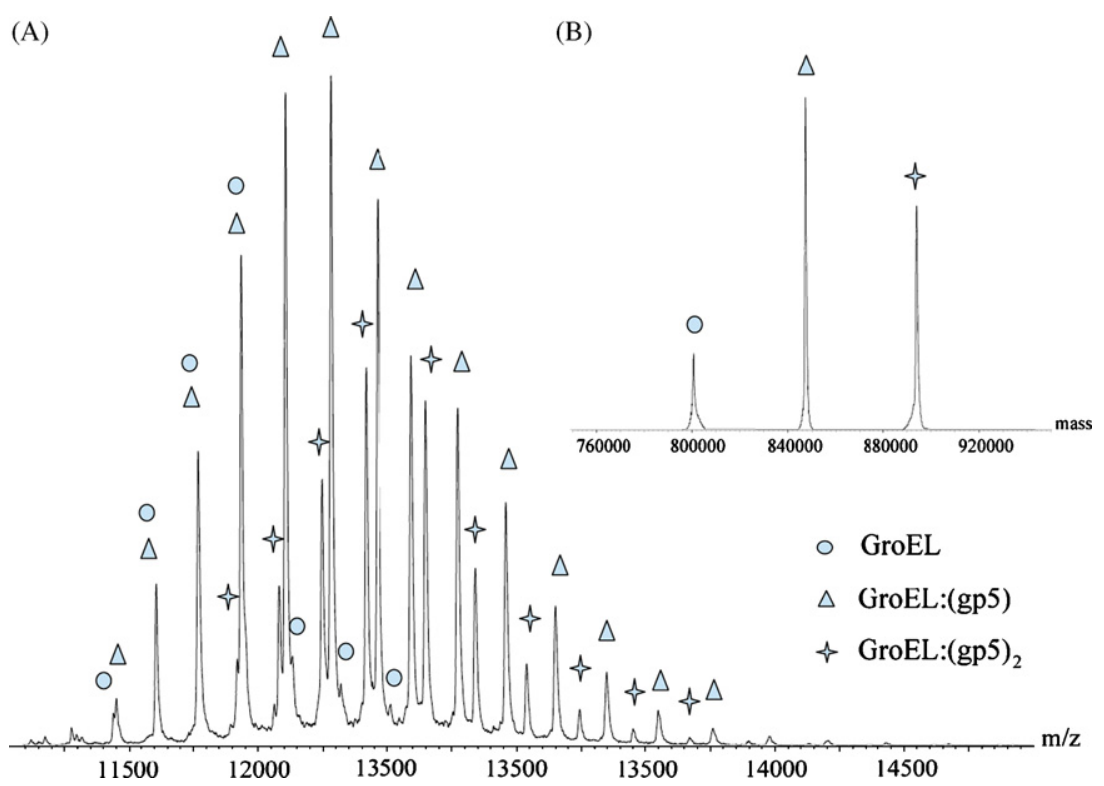

Fig. 6. ESI mass spectrum of intact GroEL gp5 substrate complexes. GroEL (14-mer) and gp5 were mixed in a 1:2 ratio. Three different charge state distributions can be observed in these spectra originating from GroEL $(\bigcirc)$, GroEL:(gp5) $(\triangle)$ and GroEL:(gp5) $(\diamond)$. In $(B)$, the corresponding convoluted zero-charge spectrum is given.

first substrate inhibits the binding of a second substrate in the opposite ring $[28,29]$. In contrast, we showed previously by macromolecular mass spectrometry that the stoichiometry of substrate binding to the chaperonin complex is dependent on the type of substrate [3,22]. For instance, the bacteriophage P22 capsid protein gp5 is able to occupy both GroEL rings simultaneously [30]. To illustrate this, an ESI mass spectrum is shown of a mixture of tetradecameric GroEL to which unfolded gp5 substrate was added in a ratio of 1:2 Fig. 6A. This rather complex mass spectrum reveals three charge state distributions, clearly originating from GroEL, GroEL:gp5 and GroEL:(gp5) as indicated by the convoluted zero-charge mass spectrum in Fig. 6B.

Next, we compared the gas-phase stability of GroEL to GroEL:gp5 and GroEL:(gp5) 2 by measuring "break-down" curves for the dissociation of these species, selectively performing tandem mass spectrometry measurements on precursor ions of the chaperone with zero, one and two substrate gp5 bound. Measurements were done with argon, krypton and xenon as collision gases. All three species revealed similar fragmentation reactions, namely the loss of one monomeric subunit at intermediate collision voltages, followed by the loss of a second GroEL monomer at higher collision voltages. Interestingly, the elimination of $57 \mathrm{kDa}$ GroEL subunits is favored over the loss of the $47 \mathrm{kDa}$ gp5 substrate, which may indicate that the substrate is still encapsulated within the GroEL ring in the gas-phase diminishing its diossciation, in line with our previous data on similar GroEL substrate complexes [22]. In Fig. 7 break-down curves are shown obtained with krypton as collision gas, but results with the other gases revealed similar effects. In Fig. 7 the breakdown curves for loss of one GroEL subunit (solid symbols) and 2 GroEL subunits (open symbols) are depicted. We evaluated break-down graphs of different charge states of all three GroEL - GroEL:gp5 - GroEL:(gp5)2 species, but always observed sim- ilar trends. The threshold for dissociation increases when one and even more when two gp5 substrate molecules are bound to the GroEL machinery. We estimated the relative thresholds for the formation of tridecameric species. For GroEL this was $0.73 \mathrm{eV}$ for GroEL:gp5 $0.87 \mathrm{eV}$ and for GroEL:(gp5) $0.91 \mathrm{eV}$, using krypton as collision gas. In the elimination of the second monomer, and thus, the formation of the dodecameric ions, the same trend is observed, with relative thresholds for elimination of the second monomer at $0.86 \mathrm{eV}$ for GroEL, GroEL:gp5 $1.01 \mathrm{eV}$ and for GroEL:(gp5) $21.06 \mathrm{eV}$ confirming that the gasphase stability of the complexes is increased by the binding of

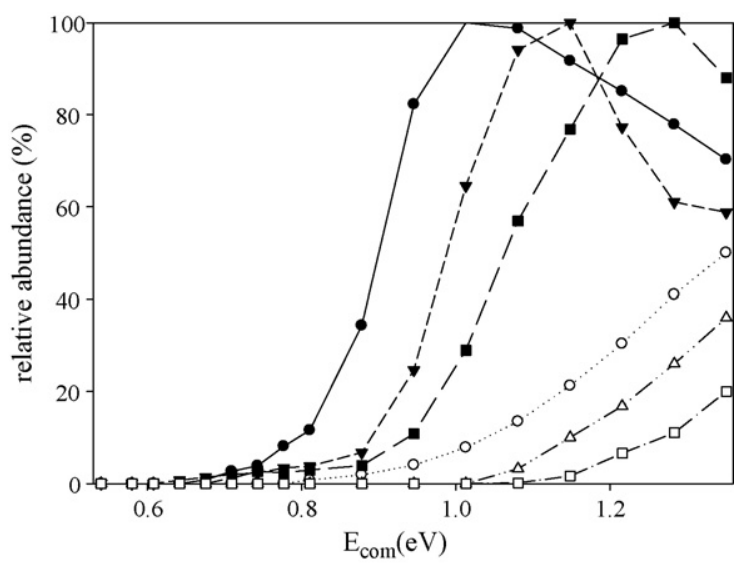

Fig. 7. Appearance curves of high mass fragment ions in the tandem mass spectra of GroEL, GroEL:(gp5) and GroEL:(gp5) . The only high mass fragment ions observed are due to the sequential loss of one and two monomeric GroEL subunits. The curves depict the observed relative ion intensities in case of GroEL $(\bigcirc, \bigcirc)$ GroEL:(gp5) $(\triangle, \mathbf{\Delta})$ and GroEL: $(g p 5)_{2}(\square, \boldsymbol{\square})$. The GroEL tridecamer fragment ions are depicted by solid symbols, whereas the dodecamer fragment ions are presented by open symbols. The tridecameric abundances are relative to their highest intensity, the dodecameric ions are relative to the corresponding tridecamer. 
substrate molecules. This result may be not that surprising as the encapsulated substrate molecules will bind multivalently to the GroEL subunits of the rings, as one substrate molecule can have numerous interactions with multiple subunits of the ring. It is interesting to note that the stabilizing effects of substrate binding, expected in solution, are mimicked in this case in the gas-phase, providing further evidence that some of the solutionphase structure of larger protein complexes may be retained in the gas-phase $[7,22,31]$.

\section{Conclusions}

Native or macromolecular electrospray ionization mass spectrometry has become a viable method to study multimeric protein complexes of increasing size and complexity, which may be used to complement other techniques in structural biology. The chemical architecture of such an assembly may be further probed by tandem mass spectrometry, which can dissect even large protein complexes of a few hundreds of $\mathrm{kDa}$ in a sequential fashion, helping to identify building blocks, and potentially the complex topology, quaternary structure and stability. Here we show that using a modified Q-ToF 1 such tandem mass spectrometry experiments may be optimized by using smaller entrance and exit orifices on the collision cell and heavier collision gas targets. With the- standard used- lighter argon as collision gas, we observed that the dissociation was not efficient, but more noticeably we observed only very low abundant high mass fragment ions in the tandem mass spectra. These effects can be largely attributed to the decreased ability to focus ions of very high $\mathrm{m} / \mathrm{z}$ before and after collisional activation, which may easily drift off-axis due to their relatively low axial velocity. Additionally, the heavier collision gasses and higher pressures may slow down the macromolecular ions more efficiently, leading to longer residence times in the collision cell, giving them more time to fragment [27]. We conclude, therefore, that the heavier xenon is the preferred collision gas for tandem mass spectrometry on very large macromolecular complexes.

\section{Acknowledgements}

We thank Saskia van der Vies for advice in purifying GroEL and Carol Teschke for purified gp5. This research was supported by the Netherlands Proteomics Centre (http://www. netherlandsproteomicscentre.nl). EvD was supported by FOM, project number 01FB12.

\section{References}

[1] J.A. Loo, Studying noncovalent protein complexes by electrospray ionization mass spectrometry, Mass Spectrom. Rev. 16 (1) (1997) 1.

[2] A.J. Heck, R.H. van den Heuvel, Investigation of intact protein complexes by mass spectrometry, Mass Spectrom. Rev. 23 (5) (2004) 368.

[3] E. van Duijn, P.J. Bakkes, R.M. Heeren, R.H. van den Heuvel, H. van Heerikhuizen, S.M. van der Vies, A.J. Heck, Monitoring macromolecular complexes involved in the chaperonin-assisted protein folding cycle by mass spectrometry, Nat. Methods 2 (5) (2005) 371.

[4] J. Borch, T.J. Jorgensen, P. Roepstorff, Mass spectrometric analysis of protein interactions, Curr. Opin. Chem. Biol. 9 (5) (2005) 509.
[5] J.L. Benesch, J.A. Aquilina, B.T. Ruotolo, F. Sobott, C.V. Robinson, Tandem mass spectrometry reveals the quaternary organization of macromolecular assemblies, Chem. Biol. 13 (6) (2006) 597.

[6] A.R. McKay, B.T. Ruotolo, L.L. Ilag, C.V. Robinson, Mass measurements of increased accuracy resolve heterogeneous populations of intact ribosomes, J. Am. Chem. Soc. 128 (35) (2006) 11433.

[7] B.T. Ruotolo, C.V. Robinson, Aspects of native proteins are retained in vacuum, Curr. Opin. Chem. Biol. 10 (5) (2006) 402.

[8] A. Poliakov, E. van Duijn, G. Lander, C.Y. Fu, J.E. Johnson, P.E. Prevelige, A.J.R. Heck, Macromolecular mass spectrometry and electron microscopy as complementary tools for investigation of the heterogeneity of bacteriophage portal assemblies, J. Struct. Biol. 157 (2) (2007) 371.

[9] S.A. Synowsky, R.H. van den Heuvel, S. Mohammed, P.W. Pijnappel, A.J. Heck, Probing genuine strong interactions and post-translational modifications in the heterogeneous yeast exosome protein complex, Mol. Cell Proteomics 5 (9) (2006) 1581.

[10] H. Hernandez, A. Dziembowski, T. Taverner, B. Seraphin, C.V. Robinson, Subunit architecture of multimeric complexes isolated directly from cells, EMBO Rep. 7 (6) (2006) 605.

[11] A.E. Ashcroft, Recent developments in electrospray ionisation mass spectrometry: noncovalently bound protein complexes, Nat. Prod. Rep. 22 (4) (2005) 452.

[12] F. Sobott, H. Hernandez, M.G. McCammon, M.A. Tito, C.V. Robinson, A tandem mass spectrometer for improved transmission and analysis of large macromolecular assemblies, Anal. Chem. 74 (6) (2002) 1402.

[13] J.A. Loo, B. Berhane, C.S. Kaddis, K.M. Wooding, Y. Xie, S.L. Kaufman, I.V. Chernushevich, Electrospray ionization mass spectrometry and ion mobility analysis of the $20 \mathrm{~S}$ proteasome complex, J. Am. Soc. Mass Spectrom. 16 (7) (2005) 998.

[14] R.H. van den Heuvel, E. van Duijn, H. Mazon, S.A. Synowsky, K. Lorenzen, C. Versluis, S.J. Brouns, D. Langridge, J. van der Oost, J. Hoyes, A.J. Heck, Improving the performance of a quadrupole time-of-flight instrument for macromolecular mass spectrometry, Anal. Chem. 78 (21) (2006) 7473.

[15] S. Sanglier, E. Leize, A. Dorsselaer, F. Zal, Comparative ESI-MS study of approximately $22 \mathrm{MDa}$ native hemocyanins from deep-sea and shore crabs: from protein oligomeric state to biotope, J. Am. Soc. Mass Spectrom. 14 (5) (2003) 419.

[16] A. Schmidt, U. Bahr, M. Karas, Influence of pressure in the first pumping stage on analyte desolvation and fragmentation in nano-ESI MS, Anal. Chem. 73 (24) (2001) 6040.

[17] N. Tahallah, M. Pinkse, C.S. Maier, A.J. Heck, The effect of the source pressure on the abundance of ions of noncovalent protein assemblies in an electrospray ionization orthogonal time-of-flight instrument, Rapid Commun. Mass Spectrom. 15 (8) (2001) 596.

[18] I.V. Chernushevich, B.A. Thomson, Collisional cooling of large ions in electrospray mass spectrometry, Anal. Chem. 76 (6) (2004) 1754.

[19] A.N. Krutchinsky, I.V. Chernushevich, V.L. Spicer, W. Ens, K.G. Standing, Collisional damping interface for an electrospray ionization time-of-flight mass spectrometer, J. Am. Soc. Mass Spectrom. 9 (6) (1998) 569.

[20] F. Sobott, C.V. Robinson, Characterising electrosprayed biomolecules using tandem-MS - the noncovalent GroEL chaperonin assembly, Int. J. Mass Spectrom. 236 (1-3) (2004) 25.

[21] M.G. McCammon, H. Hernandez, F. Sobott, C.V. Robinson, Tandem mass spectrometry defines the stoichiometry and quaternary structural arrangement of tryptophan molecules in the multiprotein complex TRAP, J. Am. Chem. Soc. 126 (19) (2004) 5950.

[22] E. van Duijn, D.A. Simmons, R.H. van den Heuvel, P.J. Bakkes, H. van Heerikhuizen, R.M. Heeren, C.V. Robinson, S.M. van der Vies, A.J. Heck, Tandem mass spectrometry of intact GroEL-substrate complexes reveals substrate-specific conformational changes in the trans ring, J. Am. Chem. Soc. 128 (14) (2006) 4694.

[23] L. Sleno, D.A. Volmer, Ion activation methods for tandem mass spectrometry, J. Mass Spectrom. 39 (10) (2004) 1091.

[24] C.M. Jones, R.L. Beardsley, A.S. Galhena, S. Dagan, G. Cheng, V.H. Wysocki, Symmetrical gas-phase dissociation of noncovalent protein complexes via surface collisions, J. Am. Chem. Soc. 128 (47) (2006) 15044. 
[25] G.J. Leggett, D. Briggs, J.C. Vickerman, Collision target-gas effects during the tandem secondary-ion mass-spectrometric analysis of polymers, J. Chem. Soc.-Faraday Trans. 86 (10) (1990) 1863.

[26] O. Curcuruto, M. Hamdan, Effects of target identity and collision energy on the fragmentation of some mass-selected protonated neuropeptides, Rapid Commun. Mass Spectrom. 7 (11) (1993) 989.

[27] M.R. Mauk, A.G. Mauk, Y.-L. Chen, D.J. Douglas, Tandem mass spectrometry of protein-protein complexes: cytochrome c-cytochrome b5, J. Am. Soc. Mass Spectrom. 13 (1) (2002) 59.

[28] J. Wang, L. Chen, Domain motions in GroEL upon binding of an oligopeptide, J. Mol. Biol. 334 (3) (2003) 489.
[29] S. Falke, M.T. Fisher, E.P. Gogol, Structural changes in GroEL effected by binding a denatured protein substrate, J. Mol. Biol. 308 (4) (2001) 569.

[30] E. van Duijn, A.J.R. Heck, S.M. Van Der Vies, Inter-ring communication allows the GroEL chaperonin complex to distinguish between different substrates, Protein Science 16 (5) (2007) 956.

[31] B.T. Ruotolo, K. Giles, I. Campuzano, A.M. Sandercock, R.H. Bateman, C.V. Robinson, Evidence for macromolecular protein rings in the absence of bulk water, Science 310 (5754) (2005) 1658. 